\title{
Estrategias para favorecer la habilidad del conteo en niños de nivel Preescolar
}

\author{
Strategies to Stimulate Counting Skills in Preschoolers
}

Julieta Fabiola Hernández Pedrasa*

Gabino Pérez Vázquez ${ }^{* *}$

\begin{abstract}
* Julieta Fabiola Hernández Pedrasa. Lic. En Educación preescolar, por la Escuela Normal del Estado de Tabasco "Rosario Ma. Gutiérrez Eskildsen" en 2010. Concluyo sus estudios de "Maestría en Docencia" en el Instituto de Educación Superior del Magisterio (IESMA) en 2015. Actualmente se desempeña como Docente y Directora Comisionada en el Jardín de Niños Beatriz Zentella de Trujillo, municipio de Centla, Tabasco.
\end{abstract}

** Gabino Pérez Vázquez. Licenciado en Ciencias de la Educación. Maestro en Administración Organizacional y Desarrollo Educativo, Doctor en Ciencias de la Educación. Profesor-investigador de la DAEA-UJAT

\section{RESUMEN:}

En la presente investigación se estudian las estrategias que docentes de la zona escolar No. 47, de Preescolar, en Centla, Tabasco, utilizan en la práctica para desarrollar el pensamiento matemático de acuerdo con el Programa de Educación Preescolar (PEP). Con la intención de recuperar estas estrategias, consideradas entre las más exitosas en el aprendizaje estudiantil, se entrevistó a 10 docentes de la zona escolar.

La recolección de datos fue recabada mediante guías de observación y entrevistas. El tipo de investigación, descriptivoexploratorio, abordó el paradigma cualitativo con enfoque constructivista: los docentes refieren que usan estrategias como trabajos individuales, en pares, por equipo y de forma grupal, dándose los mejores resultados cuando se desarrollan actividades con un aprendizaje lúdico que implique el conteo de números en un espacio de la vida cotidiana, representado en la escuela.

Cada docente utiliza y aplica de una manera particular el aprendizaje lúdico, usando diferentes materiales didácticos que sirven de apoyo para el desarrollo de competencias, no sólo matemáticas, sino de las 53 competencias más establecidas en el PEP 2011

\section{PALABRAS CLAVE:}

Estrategias didácticas, Competencias matemáticas, Educación preescolar, Contenidos matemáticos, Evaluación de competencias, Enseñanza de las matemáticas.

\section{SUMMARY}

This paper studies the strategies used in their praxis by teachers of zona escolar No. 47 at the municipality of Centla, Tabasco, México, in order to develop mathematical thinking according to the Kindergarten Education Program. The purpose is to recover those strategies deemed as the most successful in the learning of students, in order to accomplish this, 10 teachers from the referred school zone were interviewed.

Data were collected through observation guides and interviews. The research was of a descriptive-exploratory type, when questioned from the qualitative approach with a constructivist approach the teachers indicate they use strategies such as individual, peer, team and group work, and the best results are obtained when students develop activities which also include a ludic learning comprising number counting in everyday life, as represented in the school.

Each teacher uses and applies ludic learning in a very special way, using various teaching materials, which are used as support for the development of competencies, not only those of Mathematics, but also of the $54 \mathrm{com}$ petencies set out in the Kindergarten Education Program 2011. 
Perspectivas Docentes 64

Estrategias para favorecer la habilidad del conteo en niños de nivel Preescolar

Strategies to Stimulate Counting Skills in Preschoolers

Julieta Fabiola Hernández Pedrasa / Gabino Pérez Vázquez

\section{KEY WORDS:}

Teaching strategies, Mathematical competencies, Kindergarten education, Mathematics contents, Competency-based Evaluation, teaching of Mathematics.

\section{INTRODUCCIÓN}

La educación preescolar es el primer peldaño de la educación básica en México; las primeras experiencias de los pequeños en su vida escolar, las tienen dentro de los Jardines de niños. En la actualidad, en muchos países como Finlandia, Alemania, Bélgica, Francia, han adoptado el trabajo por competencias en sus programas educativos y les ha resultado eficiente; en el caso del Sistema Educativo Mexicano se empezó a trabajar con este enfoque en el nivel preescolar (2004).

"El programa se enfoca al desarrollo de competencias de las niñas y los niños que asisten a los centros de educación preescolar, y esta decisión de orden curricular tiene como finalidad principal propiciar que los alumnos integren sus aprendizajes y los utilicen en su actuar cotidiano". (SEP, 2011, p. 14).

El Programa de Educación Preescolar 2011, se rige por propósitos, estándares curriculares, principios pedagógicos y principalmente por una serie de competencias que se buscan desarrollar en los niños durante los 3 grados que conforman el nivel preescolar; éstas se encuentran organizadas en 6 campos formativos: Lenguaje y comunicación, Desarrollo personal y social, Exploración y conocimiento del mundo, Desarrollo físico y salud, Expresión y apreciación artística, y uno de los más importantes, Pensamiento matemático, que a su vez se encuentra dividido en dos aspectos, "número" y "espacio, forma y medida", donde las competencias que integran este campo se enfocan a desarrollar habilidades y nociones matemáticas acordes a la edad preescolar

Asimismo, de acuerdo con el PEP (SEP 2011, p. 12), uno de los propósitos es que los niños: "usen el razonamiento matemático en situaciones que demanden establecer relaciones de correspondencia, cantidad y ubicación entre objetos al contar, estimar, reconocer atributos, comprar y medir", y que "comprendan las relaciones entre los datos de un problema (usando) estrategias o procedimientos propios para resolverlos".
Partiendo de este propósito, considero que es necesario para todas las educadoras, tener claro cuáles son las diferentes maneras en que pueden intervenir dentro del aula para enseñar a los niños estos conocimientos, de modo que les sean fáciles de aprender, les sean interesantes y que vayan acorde a los intereses y desarrollo de los niños en edad preescolar (de 3 a 6 años)

Para dar cuenta de la realidad de los Jardines de niños, el trabajo empírico de la investigación se realizó en Tabasco, donde las escuelas de educación preescolar se encuentran organizadas en 101 zonas escolares, distribuidas en los 17 municipios que conforman la entidad. En el municipio de Centla, están localizadas 4 zonas: 46, 47, 48, 89. El lugar donde se hizo la presente investigación fueron Jardines de Niños que pertenecen a la zona 47 porque fue donde las docentes tuvieron la disposición de colaborar, al igual que es la zona donde labora el investigador. Esta zona escolar se encuentra conformada por villas, poblados y rancherías, todas ubicadas en los alrededores de la carretera que conecta al municipio de Centla con el municipio de Paraíso; esta región se caracteriza por tener cercanía a las costas del Golfo de México en Tabasco. Próximo a las escuelas se pueden encontrar pozos petroleros por lo que las localidades que la conforman tienen fácil acceso y comunicación con la cabecera municipal y con Villahermosa, la capital del Estado.

El objetivo general de la presente investigación fue proponer estrategias que desarrollen la habilidad del conteo en los niños de preescolar para lograr un aprendizaje significativo. El estudio se realizó indagando con las educadoras cómo relacionan el trabajo del campo pensamiento matemático con los planteamientos del Programa de Educación Preescolar (PEP 2011), y el conocimiento que tienen específicamente de dicho campo. Fue importante identificar cuáles son las estrategias que ponen en práctica para desarrollar las competencias matemáticas en los educandos; así, como los materiales que utilizan para llevarlas a cabo, y de todas ellas recuperar las que han considerado más exitosas en el aprendizaje de los alumnos. Tomando en cuenta estos propósitos específicos, este trabajo parte de la premisa que "la mayoría de las docentes emplean estrategias didácticas que favorecen el manejo del conteo en los niños de preescolar".

Desde esta perspectiva en la investigación se buscó favorecer el desarrollo de las competencias del campo Pensamiento matemático en su aspecto específico de número, al dar a conocer estrategias de trabajo que actualmente utilizan las 
Perspectivas Docentes 64

Estrategias para favorecer la habilidad del conteo en niños de nivel Preescolar

Strategies to Stimulate Counting Skills in Preschoolers

Julieta Fabiola Hernández Pedrasa / Gabino Pérez Vázquez

educadoras en sus aulas y que puedan éstas servir de apoyo a otras docentes de este mismo nivel para enriquecer su práctica, buscando alcanzar los propósitos y competencias establecidas en el programa de estudio con sus alumnos.

Basado en la experiencia docente, se ha notado la dificultad que muestran algunas educadoras para abordar los contenidos del campo Pensamiento matemático, en el afán de hacerlos interesantes y significativos para los niños. De allí surgió el interés de conocer cuál es la forma en que las educadoras actualmente abordan este campo del PEP y cómo favorecen las competencias establecidas en el aspecto número; en especial hacia el conocimiento de los números y el desarrollo de la habilidad del conteo, y partiendo de sus respuestas, rescatar las estrategias que resulten más útiles en sus prácticas para lograr el desarrollo de las competencias matemáticas.

Al realizar esta investigación se benefician tanto alumnos como docentes, porque así los niños podrán obtener un aprendizaje significativo a través de diversas estrategias novedosas y las docentes podrán mejorar su práctica educativa y su planeación didáctica para un mejor aprovechamiento de los alumnos; además, esto evitará el bajo rendimiento que muestran algunos niños en la actualidad al ingresar al nivel Primaria con relación al aprendizaje de las nociones numéricas.

El aprendizaje del conteo, así como de las demás nociones matemáticas, no surge de manera espontánea; a lo largo del tiempo esta problemática ha sido objeto de investigaciones: Cerda, Pérez, Ortega, Lleujo y Sanhueza (2011) llevaron a cabo la ejecución de un programa sistemático de desarrollo de la comprensión del número y el uso del conteo, el cual consistió en diversas actividades didácticas planificadas por equipo, tomando como base 6 contenidos jerarquizados con sus respectivos objetivos específicos a lograr: contar, comparar, serie numérica, ordenar números, números ordinales y descomposición numérica; estas actividades fueron comparadas con la metodología de un programa tradicional basándose fundamentalmente en contenidos y aprendizajes, obteniendo como resultado mejor rendimiento y respuesta positiva por parte de los alumnos.

Otra investigación (Micalco, 2013, p. 43) demostró que las nociones matemáticas pueden ser aprendidas por los niños a partir de su contexto y prácticas sociales en los que se encuentran inmerso, partiendo del enfoque histórico-cultural de Vygotsky, donde establece que el conocimiento es construido en un proceso de socialización entre adultos y niños en el que entran en juego determinadas formas culturales en las actividades realizadas en un grupo social.

\section{PROBLEMÁTICAS EN TORNO AL CAMPO FORMATIVO PENSAMIENTO MATEMATICO}

Favorecer el desarrollo de las competencias matemáticas en niños de educación Preescolar, está incluido en el Programa de Educación Preescolar 2011, por lo que en las aulas de preescolar, cada educadora adopta diversas estrategias didácticas para la enseñanza de sus alumnos, que resultan necesarias para enriquecer y estimular la puesta en práctica de estas competencias, de modo que estimulen en los niños el interés por el aprendizaje y la curiosidad por las matemáticas; en ese sentido, Fuenlabrada (2005, p. 57) afirma que "el logro de las competencias del pensamiento matemático, depende de una nueva concepción del aprendizaje y, por tanto, de la forma en que se de la enseñanza" ya que de no despertar este interés en la edad preescolar, en años posteriores, cuando los alumnos cursan los siguientes niveles educativos (Primaria, Secundaria, Medio superior y Superior), existe la posibilidad de que éstos no expresen curiosidad o interés por las nociones numéricas. Al contrario, los estudiantes se muestran desinteresados por los números y las matemáticas en general.

En la actualidad, en algunas aulas de nivel Preescolar, las educadoras presentan ciertas dificultades en sus prácticas como son: el no conocer o no aplicar las estrategias más favorables para estimular en los niños el desarrollo de la habilidad del conteo, de modo que sea utilizado por ellos de forma significativa en la vida cotidiana; como bien plantea Fuenlabrada (2005, p. 60). "[...] es necesario que el niño vaya interactuando con el número en situaciones donde -efectivamente- el numero tenga sentido, "el alumno plantea y resuelve problemas en situaciones que le son familiares, que implican agregar, reunir, quitar, igualar, comparar y repartir objetos. Este planteamiento va mucho más allá del trabajo con el número que tradicionalmente se realiza en la educación preescolar".

Las dificultades que presentan las educadoras son debidas a varias causas; una de ellas es la falta de disposición e iniciativa para mejorar su práctica educativa mostrando desinterés y apatía por leer, indagar, continuar estudiando, etc., lo que provoca en ellas cierto desconocimiento por las actuales investigaciones sobre el desarrollo de las competencias de los niños y las diferentes estrategias que sirven para favorecerlas en 
Perspectivas Docentes 64

Estrategias para favorecer la habilidad del conteo en niños de nivel Preescolar

Strategies to Stimulate Counting Skills in Preschoolers

Julieta Fabiola Hernández Pedrasa / Gabino Pérez Vázquez

el aula. Otra causa es el poco dominio del PEP, principalmente en las docentes con más de 15 años de servicio, quienes se excusan en que las recientes actualizaciones del programa de preescolar no les ha favorecido pues tenían mejor manejo de los antiguos programas, el 2004 o incluso el programa de 1992, lo que tiene como consecuencia que no sean trabajadas de forma adecuada el desarrollo de las competencias matemáticas.

El poco interés que tienen las docentes de preescolar (un sistema en que la mayoría de los profesores que laboran son mujeres) por innovar en el diseño de sus planeaciones didácticas, las lleva a reutilizar planeaciones de ciclos escolares anteriores lo que provoca otra de las dificultades de las educadoras ya que esto ocasiona que se aborden los contenidos matemáticos de forma tradicional, utilizando métodos y estrategias cotidianas como el copiado y las planas, delimitar los números pegando bolitas de papel, pegar semillas o colorear los números; actividades que en la actualidad resultan obsoletas y sin sentido para los niños, como bien señala Fuenlabrada (2009, p. 14): "el reconocimiento de la representación simbólica de las números se entreteje con el boleo con papel crepé para que los niños rellenen las grafías de los números o bien, pinten de colores diferentes indicaciones de la educadora". Además, a pesar de las actualizaciones constantes que se reciben en los talleres y Consejos Técnicos Escolares (CTE) durante el ciclo escolar -algunas docentes- no aplican en su práctica las nuevas estrategias que se comparten en estas sesiones y con otras escuelas para abordar el campo de pensamiento matemático del PEP, provocando que los niños en clases se muestren apáticos y desinteresados en las actividades diarias de la escuela.

Es por lo anterior, que esta investigación tuvo como finalidad conocer ¿Cuáles son las estrategias que actualmente utilizan las educadoras en la enseñanza del conteo y de qué forma realizan su intervención en las actividades matemáticas? De modo que al realizar la investigación de campo y al analizar las respuestas de las docentes, puedan darse a conocer estrategias de trabajo efectivas para favorecer las competencias del campo de pensamiento matemático.

\section{REFERENTES TEÓRICOS DE LA INVESTIGACIÓN}

La presente investigación se basó en dos disciplinas, una de ellas es la psicología, definida como la "ciencia de la conducta y de los procesos mentales" (Morris y Maistro,
2005). A través de esta disciplina pueden explicarse diferentes comportamientos que presentan los niños en edad preescolar, así como sus diferentes etapas en su pensamiento, pues esta disciplina trata de explicar aspectos del pensamiento como son los sentimientos, las percepciones y las acciones humanas que pueden tenerse ante determinadas situaciones. Una de las ramas de esta disciplina es la psicología cognitiva que se centra en el "estudio de los procesos cognitivos básicos que se identifican con las funciones clásicas de la mente: atención, percepción, memoria, aprendizaje, lenguaje, pensamiento, representación del conocimiento, categorización, etc." (Morris, y Maistro, 2005, p.5). De modo que ayudará a comprender la forma en que los niños aprenden a contar y las estrategias idóneas para intervenir en ese aprendizaje.

Otra de las disciplinas relacionada con la investigación es la pedagogía, definida por Romero (2009) como el "conjunto de saberes que se aplican a la educación como fenómeno típicamente social y específicamente humano. Es por tanto considerada una ciencia de carácter psicosocial que tiene por objeto el estudio de la educación con el fin de conocerla, analizarla y perfeccionarla". Se realiza la vinculación a esta disciplina al investigar un hecho que se efectúa en aulas de nivel Preescolar, analizar las prácticas educativas de las docentes frente a grupo y posteriormente rescatar las estrategias que resulten favorables para lograr un aprendizaje significativo del conteo en los alumnos.

Se tomó como base en esta investigación, la teoría de Lev Vygotsky, interesado en demostrar la influencia del contexto social en el proceso de aprendizaje de los niños a través de su teoría sociocultural; sostiene que el aprendizaje está influenciado de forma muy amplia por la sociedad y el contexto que rodea al pequeño, cuando se enfrentan a la necesidad de resolver problemas que se le presentan en su vida cotidiana, a la vez, estos problemas cotidianos permiten al niño un mayor manejo y utilización para desarrollar sus habilidades, "resulta muy importante la interacción que tienen los niños con su contexto social y cultural pues designa a ésta, una gran parte de la tarea del aprendizaje de los pequeños" (Bodrova \& Leong, 2014, p. 57); por lo tanto, las estrategias que se implementen dentro del aula para favorecer el uso del conteo en los niños deben planearse y trabajarse tomando en cuenta la interacción que tiene los niños con sus mismos compañeros del aula y el contexto social en el que está inmerso.

Vygotsky (citado por Kozulin, 2000 p. 36 ) asegura que "la teoría se basa en una serie de conceptos interconectados, 
Perspectivas Docentes 64

Estrategias para favorecer la habilidad del conteo en niños de nivel Preescolar

Strategies to Stimulate Counting Skills in Preschoolers

Julieta Fabiola Hernández Pedrasa / Gabino Pérez Vázquez

tales como la noción de procesos mentales superiores, a los cuales pertenecen actividades como dibujar, contar y escribir, consideradas tradicionalmente como derivadas de la inteligencia o la imaginación", estas actividades que pertenecen a los procesos mentales superiores, los describió a partir de las herramientas técnicas y psicológicas que emplean los niños para interpretar su mundo. "Las herramientas de la mente son instrumentos intelectuales que el niño utiliza para controlar su pensamiento y su conducta al igual que para ampliar sus habilidades mentales" bodrova \& Leong, (2014, p. 24). Para Vigotsky, el conteo es una de las herramientas de la mente que le sirve a los niños para resolver sus problemas y la deben adquirir en el proceso de su desarrollo cognitivo, este paso en los niños se va dando poco a poco y su estancia por el preescolar es el tiempo idóneo para que los pequeños puedan apropiarse de ella. Sin embargo, para Vigotsky todas las culturas poseen sus propias herramientas técnicas y psicológicas que se trasmite a los niños por medio de las interacciones sociales.

Muchas veces los adultos suelen confundir la acción de recitar los números de forma oral con la habilidad propia de contar, pero la definición es más compleja: "Recordar las palabras numéricas en el mismo orden, en un conjunto contar cada objeto solo una vez y entender que el número de objetos está representado por el último número que pronuncian cuando cuentan el conjunto además de comprender el sentido de contar y para qué les sirve". Nunes \& Bryant (1995, P. 36)

Otro autor que ha investigado la forma en que deben ser enseñadas las matemáticas en el nivel preescolar, es Irma Fuenlabrada (2009, P. 56), quien define contar como "un proceso donde es ineludible conocer "de memoria" la serie oral de los primeros números; es hacer corresponder el nombre de los números (según aparece en la serie) con solo un objeto de la colección que se desea cuantificar".

Favorecer el conteo en los niños, pertenece a las competencias del Programa de Educación Preescolar, actualizado en 2011 por la Reforma Integral de la Educación Básica (RIEB), mismo que define como competencia "la capacidad que una persona tiene de actuar con eficacia en cierto tipo de situaciones mediante la puesta en marcha de conocimientos, habilidades, actitudes y valores.". (SEP, 2011)

Para Fuenlabrada (2009, p.11) la palabra competencia se define como "un conjunto de capacidades que incluye conocimientos, actitudes, habilidades y destrezas que una persona logra mediante procesos de aprendizaje y que se manifiestan en su desempeño en situaciones y contextos diversos".

Por lo tanto, una competencia es la combinación de habilidades, actitudes y destrezas que van a hacer estimuladas o desarrolladas dándoles esa oportunidad a los niños en el preescolar, planteándoles problemas, propiciando la reflexión, la participación, el trabajo colaborativo, etc., no sólo aplicando actividades manuales donde el niño carece de la oportunidad de demostrar lo que sabe y lo que puede lograr hacer. Por ello, la importancia de conocer cuáles son las estrategias que resultan idóneas o recomendables para trabajar en el aula y favorecer la habilidad del conteo en los niños en edad preescolar. Se define por estrategia, el "plan general que se formula para tratar una tarea, una tarea que vuelva menos dificultosa una labor, ya que la atienden inteligentemente con método y con experiencia. (Hernández, 2003)

Las estrategias son acciones que se llevan a cabo para poder lograr entre los alumnos un aprendizaje significativo; en este sentido, otra definición que se le puede dar a una estrategia, es: "son formas de llevar a cabo metas, con conjuntos de acciones identificables, orientas a fines más amplios y generales". Rockwell (1985 P.121)

El conteo forma parte de la vida cotidiana de los niños. Se ha podido percatar de ello a lo largo de la experiencia docente en los jardines de niños, muchas veces los niños quieren saber cuántos dulces tienen o cuántos dulces necesitan para tener la misma cantidad que alguno de sus compañeros; cuántos días faltan para una fecha especial o cuánto dinero necesitan para comprar algo que desean, entre muchos otros problemas que surgen en la interacción diaria con su contexto social.

En Preescolar, regularmente los padres de familia no tienen claro este concepto ya que cuando los niños pueden recitar de memoria los números para ellos significa que sus hijos ya saben contar, sin embargo, esto corresponde a uno de los principios del conteo, propuestos por Helman y Gallistel y Helman y Mack, en la tesis "Primero principios luego capacidades". Cuando un niño aprende a contar es porque ya tiene el dominio del manejo de los 5 principios en el conteo (PEP, 2011):

Correspondencia uno a uno: contar todos los objetos de una colección una y sólo una vez, estableciendo la correspondencia entre el objeto y el número que le corresponde en la secuencia numérica. 
Perspectivas Docentes 64

Estrategias para favorecer la habilidad del conteo en niños de nivel Preescolar

Strategies to Stimulate Counting Skills in Preschoolers

Julieta Fabiola Hernández Pedrasa / Gabino Pérez Vázquez

Orden estable: contar requiere repetir los nombres de los números en el mismo orden cada vez, es decir, el orden de la serie numérica siempre es el mismo: 1, 2, 3...

Cardinalidad: comprender que el último número nombrado es el que indica cuántos objetos tiene una colección.

Abstracción: el número en una serie es independiente de cualquiera de las cualidades de los objetos que se están contando; es decir, que las reglas para contar una serie de objetos iguales son las mismas para contar una serie de objetos de distinta naturaleza canicas y piedras; zapatos, calcetines y agujetas.

Irrelevancia del orden: el orden en que se cuenten los elementos no influye para determinar cuántos objetos tiene la colección, por ejemplo, si se cuentan de derecha a izquierda o viceversa.

\section{EL CONTEXTO DE LA INVESTIGACIÓN}

De las zonas de nivel Preescolar que conforman el estado de Tabasco, esta investigación fue realizada en la zona No. 47, ubicada en el municipio de Centla, Tabasco. Conformada en su totalidad por 13 Jardines de Niños ubicados en diferentes villas, rancherías y poblados: las 5 escuelas participantes fueron elegidas por ser las de organización completa, criterio de inclusión en esta investigación; dichas escuelas están conformadas por 50 más grupos y cuentan con profesor de educación física, profesor de acompañamiento musical, en algunos casos profesor de inglés y de educación especial, una niñera, uno o dos intendentes y una directora efectiva.

Asimismo, se consideró conveniente tomar sólo a las docentes a cargo de los grupos de tercer grado ya que están integrados por niños de entre 5 y 6 años de edad, los cuales muestran cierto grado en el dominio del conteo, al haber cursado ya sus primeros dos años de educación preescolar.

Todas las escuelas cuentan con agua potable y luz eléctrica, tienen acceso y comunicación fácilmente con la cabecera municipal y con la ciudad de Villahermosa, capital de Tabasco. Las familias de los niños que asisten a dichas escuelas son consideradas en un nivel socioeconómico medio-bajo.

\section{METODOLOGÍA}

La investigación se posicionó en el paradigma cualitativo, pues "busca comprender hechos o fenómenos sociales desde su propio contexto" (Ramón y García, 2013, p.32) y realizar los estudios de ambientes singulares vinculados a la interacción social; en este caso, nos permitió conocer cuáles son las formas de trabajos de las educadoras y las estrategias que aplican en sus aulas y fuera de ellas para el aprendizaje de los alumnos, al ser ellas quienes tienen una interacción diaria en el trabajo con los niños.

I enfoque investigativo empleado fue el constructivista, (Hasimoto, E. 2005), que "trata de comprender los fenómenos o conductas desde la misma perspectiva de los involucrados en el estudio, parte de las vivencias de los sujetos; a la vez, trata de entender los significados del fenómeno para los implicados; conductas, creencias, actitudes, entre otras. Tiene como objetivos descubrir, generar, comprender el fenómeno. En este caso, se va a partir de los conocimientos que reflejen las educadoras sobre su labor docente y las estrategias de trabajo para rescatar las más significativas.

El tipo de investigación fue descriptivo-exploratorio, ya que los estudio descriptivos, de acuerdo con Hernández, Fernández, Baptista (2006, p. 101) "miden, evalúan y recolectan datos sobre diversos conceptos (variable), aspectos, dimensiones y componentes del fenómeno a investigar. En un estudio descriptivo se selecciona una serie de cuestiones y se mide o recolecta información sobre cada una de ellas para describir lo que se investiga". Por otra parte, los estudios exploratorios "sirven para familiarizarnos con fenómenos relativamente desconocidos, obtener información sobre la posibilidad de llevar a cabo una investigación más completa respecto de un contexto particular, investigar nuevos problemas, identificar conceptos o variables promisoras, establecer prioridades para investigaciones futuras, sugerir afirmaciones y postulados (Hernández, Fernández, Baptista, 2006, p. 102).

\section{INSTRUMENTOS DE RECOLECCIÓN DE DATOS}

Para la recolección de datos se utilizaron dos instrumentos: guía de observación y entrevista abierta. En lo referente a la observación "implica adentrarnos en profundidad a situaciones sociales y mantener un papel activo, así como una reflexión permanente. Estar atento a los detalles, sucesos, eventos e interacciones" (Hernández, Fernández, Baptista, 2006, p. 
Perspectivas Docentes 64

Estrategias para favorecer la habilidad del conteo en niños de nivel Preescolar

Strategies to Stimulate Counting Skills in Preschoolers

Julieta Fabiola Hernández Pedrasa / Gabino Pérez Vázquez

587), por lo tanto, siempre es importante contar con una guía de observación, que sirvió en este caso como herramienta para conducir este proceso que se llevó a cabo a través de videograbaciones, las cuales fueron realizadas por dos personas especializadas, contratadas específicamente para efectuar la tarea de grabar un día de trabajo de las diez docentes, sujetos de investigacion, dentro de su aula; durante el desarrollo de las actividades relacionadas al campo pensamiento matemático y específicamente la práctica del conteo.

La segunda herramienta fue una guía de entrevista abierta, entendida "(...) como toda conversación, centrada en el detalle, la anécdota, la fluctuación de la memoria; la entrevista nos acerca a la vida de los otros, sus creencias, su filosofía personal sus sentimientos, sus miedos" (Galindo, 1998, p. 298). La guía estuvo conformada por un total de 7 preguntas, en torno a 3 tópicos: cuatro preguntas enfocadas al programa de educación preescolar; dos preguntas a la intervención en el aula; y, una pregunta al uso de materiales didácticos. La entrevista abierta fue aplicada a las mismas docentes que fueron sujetos de investigación a través de la observación en sus clases.

\section{UNIDADES DE ANÁLISIS}

La zona escolar No. 47 de preescolar del municipio de Centla, cuenta con una plantilla de 53 docentes distribuidas en 13 Jardines de Niños que conforman la zona. Para la investigación de campo se consideró sólo a las escuelas de organización completa, criterio de inclusión en esta investigación; encontrando sólo cinco escuelas que cubrían el criterio. Asimismo, fueron incluidas sólo las docentes que tenían a su cargo grupos de tercer grado, haciendo a continuación un desglose.

Tabla 1. Escuelas consideradas en el estudio

\begin{tabular}{lcc}
\hline \multicolumn{1}{c}{ JARDIN DE NIÑOS } & No. DE DOCENTES POR ESCUELA. & DOCENTES DE 3er. GRADO \\
\hline ROSARIO CASTELLANOS & 8 DOCENTES & 3 \\
BEATRIZ ZENTELLA DE TRUJILLO & 7 DOCENTES & 2 \\
MANUEL CAMPOS PAYRO & 6 DOCENTES & 2 \\
CUAUHTEMOC & 6 DOCENTES & 2 \\
ROSARIO MA. GUTIERREZ ESKILDSEN & 5 DOCENTES & 1 \\
Total & 32 & 10 \\
\hline
\end{tabular}

Como se puede percibir, las unidades de análisis que finalmente participaron en el estudio fueron 10 docentes de cinco Jardines de niños.

\section{DISCUSIÓN DE LOS RESULTADOS}

Los resultados del estudio se presentan tomando como referente los objetivos de la investigación. En el rubro de la relación que establecen las educadoras en el trabajo del campo pensamiento matemático con los planteamientos del PEP 2011, las docentes en su mayoría muestran como evidencia un conocimiento de forma general del programa educativo, responden el fundamento de estar basado en competencias y aprendizajes esperados, hacen mención que es indispensable tener el programa al alcance para realizar sus planeaciones porque es su guía para el diseño de las situaciones didácticas en el desarrollo de las competencias que se buscan favorecer en los alumnos dependiendo el grado que cursan. Con respecto a la evaluación establecida en el programa, sólo una docente mostró el uso de una evaluación a través de rubricas donde registró las respuestas de los niños a su actividad aplicada. Las demás docentes explicaron que utilizan el diario de trabajo como una herramienta eficaz para registrar y evaluar los avances significativos que muestran los niños en clases pero no hacen uso de las diferentes herramientas de evaluación que pueden utilizarse en preescolar como lista de cotejo, guía de observación, escala de actitudes, registro anecdótico, etc.

En el dominio que muestran las educadoras del conocimiento en relación al campo pensamiento matemático y el uso de términos matemáticos en clases, las preguntas no fueron totalmente acertadas en los cuestionamientos sobre los propósitos específicos del campo, los principios del conteo, la resolución de problemas, suelen confundir algunos términos 
Perspectivas Docentes 64

Estrategias para favorecer la habilidad del conteo en niños de nivel Preescolar

Strategies to Stimulate Counting Skills in Preschoolers

Julieta Fabiola Hernández Pedrasa / Gabino Pérez Vázquez

o les falta mayor claridad en sus definiciones, lo que tiene como consecuencia que al llevar a cabo sus clases -como se pudo corroborar a través de las vídeograbaciones- usan muy poco los términos matemáticos como: igualdad, desigualdad, contar uno a uno, llevar un orden estable, contar una colección, representar objetos, orden ascendente y descendente etc. Al sustituirlos por términos coloquiales como empatados, uno por uno, contar de atrás para adelante y de adelante para atrás, contar de corrido, etc.

Otro de los objetivos de investigación fue la de describir las estrategias didácticas que emplean las educadoras dentro del aula, donde también se toma en cuenta su intervención en la ejecución de las actividades, control de grupo, tono de voz, ayuda a los niños que muestran dudas o dificultades. En este caso, de las 10 docentes solo una no mostró un dominio total en el control del grupo y no se consideró la ejecución de la actividad completamente exitosa al observar que los niños no respondían a las indicaciones que ella les daba; el resto de las docentes mostró un buen control de grupo, un tono de voz adecuado. Igualmente, las 10 docentes realizaron intervenciones pertinentes durante el desarrollo de las actividades, acercándose y ayudando a los niños que mostraban dificultades o despejando algunas dudas que expresaban. En cuanto a las estrategias implementadas, se pudieron observar "in situ" diferentes formas de trabajo para organizar a los niños: trabajos individuales, en pares, por equipos y de forma grupal, otras estrategias para el aprendizaje de los números y la puesta en práctica del conteo, fue la que aplicaron 8 de las 10 docentes; al entrar al salón de clases e iniciar la jornada escolar, contar a las niñas y niños asistentes de ese día, colocar la cantidad y representarlos a través de dibujos, de modo que los niños pongan en práctica los principios del conteo, en este sentido, 2 docentes ocuparon el pase de lista para que los niños fuesen mencionando los números del 1 al 10 en vez de utilizar la palabra "presente", 4 docentes utilizan canciones donde hace mención de la serie numérica oral del 1 al 10 y a su vez induce a los niños al conteo, dentro de las situaciones didácticas centrales plasmadas en la planeación, 8 docentes utilizan "el juego" como estrategia principal para el desarrollo de las actividades, lo que permite a los niños preescolares involucrarse de forma más activa en ellas, manifestándolo a través de diversas formas como: juegos con dados en equipos... cada niño debía tomar la cantidad de cereales que marcaban los puntos en los dados, al finalizar ganaba el niño que más cereales había juntado para después comérselos; juegos tradicionales en el patio pedagógico como el avión, organizados en equipos jugaron tirando una fichita y tratando de atinar a cada recuadro, llevando el numero consecutivo del 1 al 10. Encestando tapas en un cartón con pequeños huecos (cono de huevo), organizados en equipos cada integrante tenía 4 tapas y por turnos debían pasar a encestarla, finalmente ganaba el equipo que lograba encestar más tapas. Buscando la pareja, organizados en dos equipos, uno tenía los números y el otro la cantidad pegados en una hoja en el pecho, debían encontrarse la pareja correcta numerocantidad, perdían la última pareja en encontrarse. Juego de lotería con números, donde la docente enseña la carta dice el número y los niños lo localizan en su cartilla para marcarlo con una ficha si lo tienen. Juego con un tren de 10 vagones, hecho con material reciclable y llevado de casa, en cada vagón debía colocar el niño la cantidad de objetos según el número indicado en cada uno. Insertando palitos en una botella de plástico llena de agujeros, en parejas los niños debían colocar tapas encima de los palitos e ir sacándolos con cuidado de modo que se cayeran la menor cantidad de tapas dentro de la botella. 2 de las 10 docentes, utilizaron métodos tradicionales que no dejan de ser de igual forma provechosos para los niños como recortar de revistas o libros los números para pegarlos de forma ordenada. En sus respuestas al cuestionario, hacen mención del uso del juego para el diseño de sus situaciones didácticas en diferentes complejidades y sentidos.

Un hallazgo significativo fue el hecho que las educadoras que cuentan con una mayor edad en años de servicio son quienes no muestran la misma innovación en su práctica como lo hicieron las maestras con pocos años en la práctica.

Por otra parte se identificó a través de las vídeograbaciones, los materiales didácticos utilizados por las educadoras para el trabajo de las competencias matemáticas, los cuales son los didácticos prefabricados para el uso en el aula como dados, pelotas, loterías, palitos de madera, tapas, hojas, lápices de colores, tijeras; sin embargo, 2 docentes ocuparon material reciclable como fue conos de huevo y botellas de plástico, lo que significa que cualquier tipo de recurso puede ser utilizado con un fin educativo cuando la educadora le da un sentido didáctico y a través de ellos logra un aprendizaje en los niños. 3 docentes ocuparon cereal de colores y sólo una docente ocupó el libro "mi álbum preescolar", que es un recurso de apoyo enviado por la SEP para uso de todos los niños que cursan el nivel Preescolar en México.

A través de las encuestas se rescataron otros materiales que también utilizan las docentes como: dominó, pelotas, balanzas, tangram, rompecabezas, monedas 0 billetes didácticos, tarjetas, tableros, memoramas, barajas, libros, relojes, calendarios, laminas, conos, fichas de ensamble, plastilina, tragabolas, boliches, cuentos, etc. También se encontró el 
Perspectivas Docentes 64

Estrategias para favorecer la habilidad del conteo en niños de nivel Preescolar

Strategies to Stimulate Counting Skills in Preschoolers

Julieta Fabiola Hernández Pedrasa / Gabino Pérez Vázquez

empleo de materiales de rehúso como: las botellas de plástico, conos de huevo, cartón, bolsas, semillas, envolturas, pastas, etc.

Finalmente, se buscó rescatar las estrategias exitosas que han tenido las docentes en el trabajo del campo pensamiento matemático y específicamente favoreciendo el uso del conteo, donde señalan prácticamente todas las educadoras que utilizan el juego para abordar dichos contenidos. Una docente explicó que una modalidad de trabajo en el programa de educación preescolar es el uso de "rincones" donde a los niños dentro o fuera del aula se les escenifica un espacio de la vida cotidiana, ella llevó a cabo "el rincón de la tiendita" donde se armó una tienda con cajas y botes vacíos de productos reales, los niños tuvieron la oportunidad de jugar el papel de vendedor y comprador, al tener monedas y billetes didácticos y realizar cuentas para comprar y vender, poniendo en juego el uso del conteo y el conocimiento de los números, considerándola una de las más exitosas pero que implica un mayor esfuerzo y la participación de los padres de familia para poder transformar el salón y pueda ser de gran impacto para los niños.

\section{CONCLUSIÓN}

Lograr que los niños obtengan el aprendizaje de las nociones matemáticas resulta un largo proceso pero principalmente una gran labor para todas las educadoras que imparten educación preescolar a niños de 3 a 6 años. Contar no significa recitar los números en orden estable sino es una habilidad muy compleja, que en educación Preescolar se empieza a desarrollar en los pequeños.

En el caso de la presente investigación, en las líneas siguientes, se da cuenta de los hallazgos más significativos con respecto a las estrategias que emplean las educadoras para favorecer la habilidad del conteo en niños de nivel preescolar, los resultados se muestran con base a los objetivos planteados.

Con respecto a los saberes sobre el Programa de Educación Preescolar 2011, las docentes muestran un conocimiento general del PEP pero no un dominio total del mismo, pues hacen mención de algunos conceptos clave manejados en el programa, sin embargo, no tienen una definición clara de estos, ni de sus componentes.

Con respecto a la evaluación de los aprendizajes, en su mayoría las educadoras no muestran un uso variado de las diferentes herramientas de evaluación, aun cuando el Programa Educativo hace referencia a la utilización de estas alternativas como una forma de registrar los avances en los alumnos; la evaluación en el nivel Preescolar se realiza de forma cualitativa por lo que se requiere de llevar un registro individual durante todo el ciclo escolar.

Asimismo, se encontró que las docentes muestran poco o nulo uso de los términos matemáticos en la ejecución de sus clases, regularmente se remiten al uso del lenguaje coloquial, por ejemplo "contar de corrido" "contar de adelante para atrás" "empatados", cuando que en este nivel educativo los niños deben empezar a familiarizarse con los términos correctos como son "contar en orden estable" "contar de forma ascendente 0 descendente" etc.

Durante las clases, se observó que las adecuadoras mostraban dominio del grupo, a la vez se percibían atentas a las dudas y dificultades que presentaban los niños en el desarrollo de las actividades. Por otra parte, el juego es utilizado como la estrategia didáctica principal para la enseñanza del conteo, cada educadora de acuerdo con sus necesidades y a la innovación en sus planeaciones le da diferente sentido, los niños son organizados según el juego a implementar. Una docente, utilizó la modalidad de trabajo "el rincón" donde escenífco una tiendita y los niños a través del juego, se involucraron siendo "vendedores o compradores"; practicando el uso del conteo al "comprar o vender" productos de una tienda, utilizando monedas y billetes didácticos.

Los materiales didácticos utilizados en las aulas, regularmente son los prefabricados; no obstante, dos docentes mostraron creatividad al darle sentido pedagógico a materiales reciclables como botellas de plástico y cartón, lo que significa un apoyo a la economía de los padres de familia; y, a la vez se fomenta la preservación del medio ambiente.

A partir de los hallazgos mencionados, la hipótesis de investigación "la mayoría de las docentes emplean estrategias didácticas que favorecen el manejo del conteo en los niños de preescolar" se acepta, en virtud que cada docente utiliza y aplica de manera particular diferentes estrategias didácticas, implementando, a la vez, el uso de diversos materiales educativos; los cuales favorecen el desarrollo de las competencias, no sólo matemáticas sino de las 53 competencias más establecidas en PEP 2011.

Solo resta señalar que los resultados de esta investigacion 
Perspectivas Docentes 64

Estrategias para favorecer la habilidad del conteo en niños de nivel Preescolar

Strategies to Stimulate Counting Skills in Preschoolers

Julieta Fabiola Hernández Pedrasa / Gabino Pérez Vázquez

quedan a disposicion de la Secretaria de Educación del Estado de Tabasco (SETAB) y a todos los docentes e investigadores interesadas en esta tematica.

\section{REFERENCIAS BIBLIOGRÁFICAS}

Bodrova E. \& Leong D. (2004). Herramientas de la mente: el aprendizaje en la infancia desde la perspectiva de vygotsky. México. Editorial Pearson.

Cerda G. Pérez C. Ortega R. Lleujo M. y Sanhueza L. (2011) Fortalecimiento de competencias matemáticas tempranas en preescolares, un estudio chileno. Psychology, Society, \& Education. 3, PP. 23-39 consultado en file://I rtalecimientoDeCompetenciasMatematicasTempranas E-3738121.pdf

Elsie Rockwell. (1985) Ser maestro, estudios sobre el trabajo docente. México. SEP.

Fuenlabrada I. (2005) El programa de Educación Preescolar 2004: una nueva visión sobre las matemáticas en el jardín de niños. Cero en conducta. 51 PP. 5-74

Fuenlabrada I. (2009). ¿Hasta el 100?... jno! ¿y las cuentas?... tampoco, entonces... ¿Qué?. Mexico, SEP.

Hernández R. (2003). Mediación en el aula. Recursos, estrategias y técnicas didácticos. Cuadernos para la enseñanza del español l. costa rica. EUNED
Kozulin A. (2000) Instrumentos psicológicos: la educación desde una perspectiva sociocultural, Barcelona. Ediciones Paidós Ibérica,

Micalco M. (2014) Aprender matemáticas sin matemáticas. Una propuesta de intervención basada en las prácticas sociales del contexto de los estudiantes. Educando para educar. 26. PP. 43-55

Morris G, Maistro A. y Ortiz M. (2005) Introducción a la psicología. México. Pearson Educación.

Nunes T. \& Bryant P. (1995) Las matemáticas y su aplicación: La perspectiva del niño. MEXICO. Siglo XXI editores.

Ramón P. y García V. (2013). Guía para la Elaboración de Proyectos en Investigación Educativa. MEXICO. CONACYTUJAT.

Rodríguez B. Hoffmann M. Mackedanz F. (2011) Cómo investigar cualitativamente. Entrevista y Cuestionario. Contribuciones a las Ciencias Sociales, consultado en www. eumed.net/rev/cccss/11/

Sep, (2011). Programa de Educación Preescolar. México.

Taylor,R. y Bogdan (2000) Introducción a los métodos cualitativos de investigación. Barcelona. Ediciones Paidós Ibérica 